\title{
Comparison of Two Gas Injection Methods for Generating Bubbles in a T-Junction
}

\author{
S. Arias
}

\begin{abstract}
In this work, we compare the performance of two different methods for generating bubbles in conditions relevant to microgravity. A T-junction formed by two cylindrical (1 mm of internal diameter) and perpendicular channels is used to generate trains of non-wetting bubbles. Air and distilled water are used as dispersed and continuous phases, respectively. The difference between both methods lies in the strategy used to inject the fluids. In one case (here referred to as TJ-A configuration), the gas is injected into the side channel. In the other case (referred to as TJ-B), the gas is injected into the main channel. Bubble size dispersion is analyzed and quantified with the polydispersity index. The increase of the capillary number (based on the continuous phase) has shown a strong influence on the regularity of the bubble generation process. The TJ-A method can produce monodisperse bubbles at higher capillary numbers than configuration TJ-B. The limits of monodispersity, as far as the capillary number is concerned, are studied. These limits determine the optimal operating range of the T-junction for generating highly monodisperse bubbles. Beyond these limits, polydispersity increases, although much more abruptly in TJ-B than in TJ-A. The bubble generation frequency is also studied, with special attention to the two main regimes that characterize the frequency, i.e. the linear regime (at very low gas flow rate) and the saturation regime (at high gas flow rates). A new dimensionless expression of the bubble generation frequency is proposed in this work.
\end{abstract}

Keywords Microgravity - Two-phase flow · Bubble formation · Microchannel T-junction · Monodisperse bubbles

\section{Introduction}

Due to their promising commercial applications, two-phase flows have claimed attention over the past two decades. Applications for industries as diverse as food,

S. Arias (Corresponding Author)

Escola d'Enginyeria de Telecomunicació i Aeroespacial de Castelldefels, Universitat Politècnica de Catalunya, c/ Esteve Terradas 5, 08860 Castelldefels, Barcelona (Spain)

Tel.: +34 934134129

E-mail: santiago.arias@upc.edu

ORCID: 0000-0001-9233-0178 
pharmaceutical, medicine, cosmetic or personal care have been suggested. When two-phase flows are generated inside channels with small internal dimensions (of the order of $1 \mathrm{~mm}$ or less), gravitational forces usually play a negligible role in comparison with dominant forces (often viscous and interfacial forces). Under these circumstances, the fluids can thus be considered in conditions relevant to microgravity. This situation offers an ideal scenario to gain a better understanding of the physical interaction between the two phases. Furthermore, research on two-phase flows in small channels generates knowledge that could be applied in improving technology of space-based platforms. Some examples can be found in life support systems for human space exploration, space thermal management systems and propulsion systems.

The formation of controlled two-phase flows is a complex physical problem governed by a wide range of variables, such as the number of involved channels (usually two, but not necessarily), the channel internal geometry (triangular, square, rectangular, circular section, etc), the relative position between channels (perpendicular, parallel, with variable orientation), the channel physical properties (surface wettability, roughness, contact angle), the type of channels used for both phases (all channels can be equal or could have different dimensions and physical characteristics), fluids properties (density, viscosity, surface tension) and the operating settings (injection flow rates, fluids injection configuration, injection pressures, outlet pressure, temperature, etc). For an accurate control on the formation of two-phase flows, a better knowledge of the influence of all these variables is mandatory. Despite all the efforts made in recent years, many questions concerning the formation of two-phase flows, and the influence of some of these variable, remain open.

There are several passive methods to generate controlled two-phase flows, of which the most frequently used are the co-flowing, flow-focusing and T-junctions. The three of them have been extensively studied, being used to generate both liquid-liquid and gas-liquid two-phase flows (Utada et al., 2007; Zhao and Middelberg, 2011; Fu and Ma, 2015). Each method presents its own peculiarities. A good understanding of their performances enables selection of the best method for any specific application. In this work, we focus on the T-junction, one of the most popular geometries used for generating bubbles/droplets, due to its usability, high bubbles/droplets generation rate and small size dispersion (Thorsen et al., 2001; Nisisako et al., 2002; Van der Graaf et al., 2005; Xu et al., 2008; Guo and Chen, 2009; Malekzadeh and Roohi, 2015).

T-junctions are typically formed by the main channel and the side perpendicular channel. There are two main configurations for the injection of fluids in T-junctions. In the first one, the continuous phase is injected from the main channel, and the dispersed one is introduced from the side channel. In the second one, the inlets are exchanged, being the continuous phase injected into the side channel and the dispersed phase into the main channel. Of these two configurations, the first one is the most commonly used and thus, the one that has been most widely studied. In this paper, we refer to the first configuration as TJ-A and to the second one as TJ-B. Fig. 1 shows an example of both configurations.

Most of the time, researchers use just one method to generate two-phase flows, and the comparison between several methods (co-flowing, flow-focusing and $\mathrm{T}$ junctions), or fluids injection strategies (for example, between TJ-A and TJ-B), has been little studied. Nevertheless, some stimulating examples in this regard can 


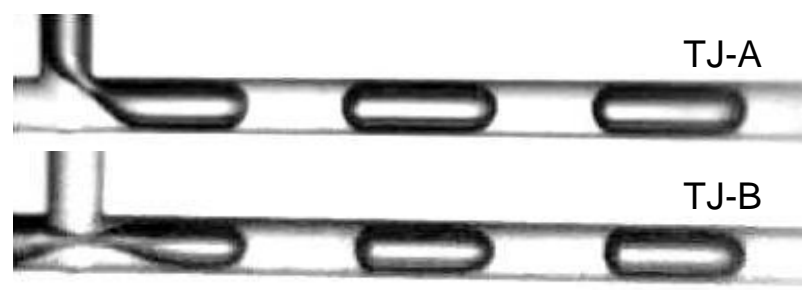

Fig. 1 Example of bubbles being generated with TJ-A and TJ-B configurations

be found in the literature. Xu et al. (2006) used T-junction microfluidic devices to prepare monodisperse water-in-oil and oil-in-water emulsions. They introduced oil from the main channel and water from the side one. By changing the wetting properties of the microchannel walls with surfactants, the authors forced the water (or the oil) to be the dispersed phase. When water behaved as the dispersed phase (the T-junction working in TJ-A mode), water-in-oil monodisperse emulsions were generated. When oil behaved as the dispersed phase (the T-junction working in TJB mode in this second case), oil-in-water monodisperse emulsions were generated. Under similar experimental conditions, the authors found that oil droplets were slightly larger than water droplets.

Abate et al. (2009) studied the impact of the inlet channel geometry on microfluidic drop formation. They used water and oil as discontinuous and continuous phases, respectively. The authors found that the fluid injection strategy strongly influenced the droplet size, as well as the water/oil flow rate ratios over which monodisperse emulsions could be obtained. Whereas T-junction devices formed monodisperse emulsions at low and moderate capillary numbers, flow-focusing drop makers did so at moderate and high capillary numbers. The authors also compared methods TJ-A and TJ-B and found that at low capillary numbers, droplets generated with TJ-A were larger than those formed with TJ-B, but droplets sizes were quite similar at moderate capillary numbers.

Tan et al. (2009) focused on the TJ-B configuration, but changing the angle between the main and side channels (that ranged from $30^{\circ}$ to $150^{\circ}$ ). They injected an aqueous solution with glycerol into the side channel and air into the main channel of a the microfluidic device. The authors found that bubble size relied on several influential factors including the gas/liquid flow rates, the physical properties of the continuous phase, and the angle between the entrance channels. Under the same circumstances, bubble sizes reached minimum values at an intersection angle of $90^{\circ}$, i.e. a pure TJ-B configuration. They derived an expression relating the angle between the channels to the bubble size. Carrier et al. (2014) investigated the formation of dispersed silicon oil droplets in water in T-junction microfluidic devices. They found no significant effect of the flow injection configurations (between TJ-A and TJ-B) on the droplet size (although the droplets sizes were different). However, satellite droplets (small droplets that form sometimes close to the main droplets) did seem to be sensitive to the flows injection method.

Possibly, the most important conclusion of all these studies (and similar works found in the related literature) seems to be that the fluids injection method is a key factor of the formation of bubbles/droplets in T-junctions. Changes in the fluids injection strategy are expected to produce significant changes in the bubble size, 
the bubble size dispersion and the bubble generation frequency. For such a reason, we present in this work a direct comparison between the two most used methods of operating a T-junction, TJ-A and TJ-B, aiming to tackle these issues. Section 2 briefly describes the problem statement and methodology, identifying the relevant dimensionless numbers that characterize the bubble generation process. Section 3 presents the results of this work, whereas the main conclusions are outlined in Section 4.

\section{Problem statement and methodology}

Experiments were performed with a T-junction bubble generator. The T-junction was formed with two perpendicular cylindrical channels of $\phi_{c}=1 \mathrm{~mm}$ each one, where $\phi_{c}$ is the channel internal diameter. This cylindrical geometry differs from the usual T-junctions with rectangular (or square) section mostly used in microfluidic devices. Our T-junction produced trains of non-wetting bubbles by mixing air (dispersed phase) and distilled water (continuous phase). Bubbles were thus produced by the crossflow action of the liquid over the gas. Two different gas injection methods were tested. In the first one, the distilled water was injected through the central inlet, corresponding to the main channel (TJ-A configuration). In the second method, the distilled water was injected through the side channel (TJ-B configuration).

Air and distilled water were considered as incompressible. Isothermal conditions at a room temperature of $25^{\circ} \mathrm{C}$ were also considered. Henceforth, subscripts $G$ and $L$ stand for gas and liquid, respectively. Standard values at $25^{\circ} \mathrm{C}$ were thus used for viscosity $\left(\mu_{G}=10^{-5}\right.$ Pa.s and $\mu_{L}=10^{-3}$ Pa.s $)$, density $\left(\rho_{G}=\right.$ $1.225 \mathrm{~kg} / \mathrm{m}^{3}$ and $\left.\rho_{L}=10^{3} \mathrm{~kg} / \mathrm{m}^{3}\right)$ and gas-liquid surface tension $(\sigma=0.072 \mathrm{~N} / \mathrm{m})$.

Experiments were performed by maintaining the volumetric gas flow rate $\left(Q_{G}\right)$ at a constant value while increasing the volumetric liquid flow rate $\left(Q_{L}\right)$. Gas and liquid superficial velocities were defined as $U_{S G}=Q_{G} / A$ and $U_{S L}=Q_{L} / A$, respectively, where $A$ is the channel cross-section area. The mixture superficial velocity $\left(U_{M}\right)$ is the sum of both superficial velocities, $U_{S G}$ and $U_{S L}$. The range of values of these superficial velocities is presented in table 1 .

Images were taken using a high-speed camera recording at 4000 frames per second (f.p.s). The time required to form and detach a single bubble (denoted from now on with the subscript $i$ ) was computed as $T_{i}=$ \#frames $/ 4000$ f.p.s., being \# frames the total number of frames required to generate that bubble. The bubble generation frequency (for a single bubble) was then calculated as $f_{i}=1 / T_{i}$.

The bubble volume of a single bubble $\left(V_{B_{i}}\right)$ was estimated taking into account the simple mass conservation equation $Q_{G}=f_{i} \cdot V_{B_{i}}$, i.e. $V_{B_{i}}=Q_{G} \cdot T_{i}$. The bubble equivalent diameter of a single non-spherical bubble $\left(\phi_{B_{i}}\right)$ was defined as the diameter of a spherical bubble with the same volume, i.e. $V_{B_{i}}=\pi \cdot \phi_{B_{i}}{ }^{3} / 6$. Normalizing the bubble volume (with $A \cdot \phi_{c}$ ) and the bubble equivalent diameter (with $\phi_{c}$ ), the following expression can be obtained:

$$
\bar{\phi}_{B_{i}}=\left(\frac{3}{2} \bar{V}_{B_{i}}\right)^{1 / 3}
$$


Table 1 Characteristic experimental values

\begin{tabular}{|c|c|c|c|c|c|c|c|}
\hline & $\begin{array}{l}U_{S G} \\
{[\mathrm{~m} / \mathrm{s}]}\end{array}$ & $\begin{array}{l}U_{S L} \\
{[\mathrm{~m} / \mathrm{s}]}\end{array}$ & $\begin{array}{l}U_{M} \\
{[\mathrm{~m} / \mathrm{s}]}\end{array}$ & $B o$ & $W e$ & $R e$ & $\begin{array}{l}C a \\
{\left[10^{-3}\right]}\end{array}$ \\
\hline $\begin{array}{l}\text { Minimum } \\
\text { value }\end{array}$ & 0.04 & 0.11 & 0.15 & 0.14 & $3.8 \cdot 10^{-4}$ & 149 & 1.5 \\
\hline $\begin{array}{l}\text { Maximum } \\
\text { value }\end{array}$ & 1.09 & 1.59 & 2.68 & 0.14 & 0.12 & 2682 & 22.1 \\
\hline
\end{tabular}

The bubble generation frequency $f$, the bubble volume $V_{B}$ and the bubble equivalent diameter $\phi_{B}$ of each pair $U_{S G}-U_{S L}$ were estimated as the average values of $f_{i}, V_{B_{i}}$ and $\phi_{B_{i}}$, respectively, over a large enough sample of bubbles.

\subsection{Dimensionless numbers}

The dimensionless numbers that characterize the current problem are described next. The Bond number $(B o)$ was defined as $B o=\rho_{L} g \phi_{c}^{2} / \sigma$, being $g$ the gravitational acceleration. The Weber number $(W e)$ was defined as $W e=\rho_{G} \phi_{c} U_{M}^{2} / \sigma$. The Reynolds number $(R e)$ was defined as $R e=\rho_{L} \phi_{c} U_{M} / \mu_{L}$. Finally, the capillary number $(\mathrm{Ca})$, based on the velocity of the continuous phase, was defined as $C a=\mu_{L} U_{S L} / \sigma$. Note that the fluids physical properties are constant along this work, and thus, the increase of $U_{S L}$ leads to the increase of $C a$.

As shown in table 1 , the value of the Bond number was small enough to consider that gravity played a negligible role (in comparison with interfacial forces) during the bubble formation process $(B o<0.29$, (Suo and Griffith, 1964)). Thus, the results of this work can be assumed as gravity independent and potentially useful for the field of microgravity. The Weber number was also small enough to consider that interfacial forces overcame inertial forces ( $W e \leq 2$, (Rezkallah, 1996)). Concerning the Reynolds number, most of the experiments took place under laminar conditions, and thus, the viscous forces exerted by the continuous phase dominated over the inertial forces. Therefore, and accordingly to the values of $B o, W e$ and $R e$ shown in table 1, the bubble generation process analyzed in this work can be considered as mainly controlled by the competition between viscous and interfacial forces. The capillary number, which describes the relative effect between these two main forces, becomes thus a key dimensionless number of this analysis. In this study, $C a$ ranged from low (order $10^{-3}$ ) to moderate values (order $10^{-2}$ ).

\section{Results and discussion}

Figs. 2 and 3 show examples of trains of bubbles produced with the TJ-A and TJ-B methods, respectively. The values of $U_{S G}$ and $C a$ are indicated in the figures. As the capillary number increases, the bubble volume decreases, which provokes the corresponding increase of the bubble generation frequency. Subsection 3.1 provides more information on the bubble volume (through the bubble equivalent diameter), while additional information on the bubble generation frequency can be found in subsection 3.2. 
A quick view of figs. 2 and 3 reveals that the capillary number significantly affects the gas breakup mechanism in both configurations. At low $C a$ (see for example $C a=1.5 \cdot 10^{-3}$ in figs. $2-3$ ), the entering gas tends to fill the main channel, blocking it. This blockage causes a pressure buildup (upstream the forming bubble) that pushes the gas downstream, provoking the gas breakup and the generation of a new bubble (fig. 11 shows an example of a complete bubble formation cycle in those circumstances). This gas breakup is commonly referred to as squeezing mechanism, and it is mainly dominated by interfacial forces and the effects of the confinement of geometry of the main channel (Garstecki et al., 2006; De Menech et al., 2008). At moderate $C a$ (see for example $C a=11.8 \cdot 10^{-3}$ in fig. 2-3), the viscous forces exerted by the liquid plays a more dominant role, shearing off the emerging bubble and preventing the gas to fill the main channel. The formation of bubbles depends increasingly on the liquid shearing forces and to a lesser extent on the pressure buildup upstream the bubble. A gap appears between the forming bubble and the wall of the main channel, the pressure buildup releases and the squeezing mechanism decays (fig. 12 shows an example of a complete bubble formation cycle in those circumstances). This gas breakup is typically called dripping mechanism (Garstecki et al., 2006; De Menech et al., 2008). In the range of capillary numbers analyzed in this work, both gas breakup mechanisms are present in the formation of bubbles in the TJ-A and TJ-B methods. Subsection 3.3 provides detailed information in this regard.

As just explained, the bubble generation process is strongly affected by the capillary number, which consequently has also a big impact on the characteristics of the bubbles (size, volume, geometry, etc), as well as on the regularity of the process itself. Indeed, the increase in $C a$ (for a given $U_{S G}$ ) leads to a loss of regularity in the bubble formation process, with the consequent increase in bubble size dispersion. This increase of the irregularity can also be seen in parameters other than the bubble volume, such as the separation between bubbles, which becomes also irregular. When regularity is lost, bubbles cease to be monodisperse. That loss of regularity becomes noticeable from value $C a=13.3 \cdot 10^{-3}$ (fig. 2) and $C a=8.8 \cdot 10^{-3}$ (fig. 3). The following subsection provides further quantitative information on the bubble size dispersion.

\subsection{Bubble size dispersion}

As stated above, the gas injection method and the capillary number have a strong influence on the regularity of the bubble generation process. This section focuses on the bubble equivalent diameter and its variability in connection to the capillary number. Fig. 4 shows the normalized bubble equivalent diameter as a function of $\mathrm{Ca}$ for several gas superficial velocities. In this figure, as well as in all the graphs of the following subsections, solid and empty points are always used for the TJ-A and TJ-B configurations, respectively. The same behavior of $\bar{\phi}_{B}$ (as a function of $C a$ ) was observed for all values of $U_{S G}$ studied in this work. For simplicity, only three representative values of the gas superficial velocity were plotted on fig. 4 for each gas injection configuration.

The standard deviation of the normalized bubble equivalent diameter was calculated for each experimental value and plotted as error bars. Error bars smaller than the corresponding symbols were not included, i.e. for $C a$ values below 13.3 . 


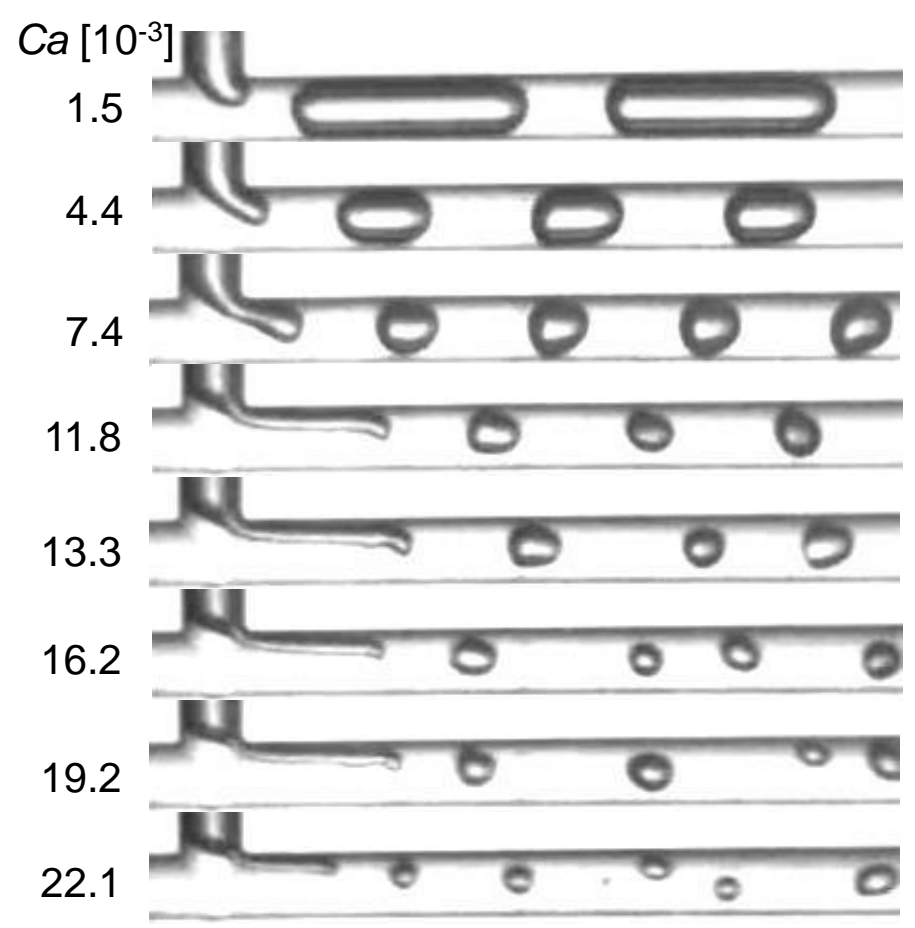

Fig. 2 TJ-A configuration: trains of bubbles at several capillary numbers for $U_{S G}=0.17 \mathrm{~m} / \mathrm{s}$

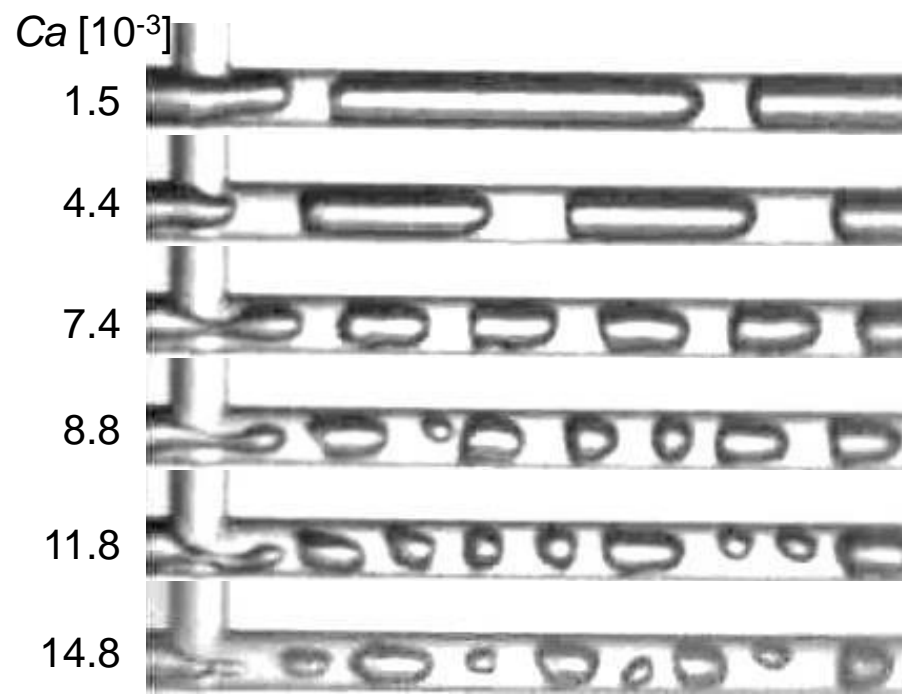

Fig. 3 TJ-B configuration: trains of bubbles at several capillary numbers for $U_{S G}=0.5 \mathrm{~m} / \mathrm{s}$ 


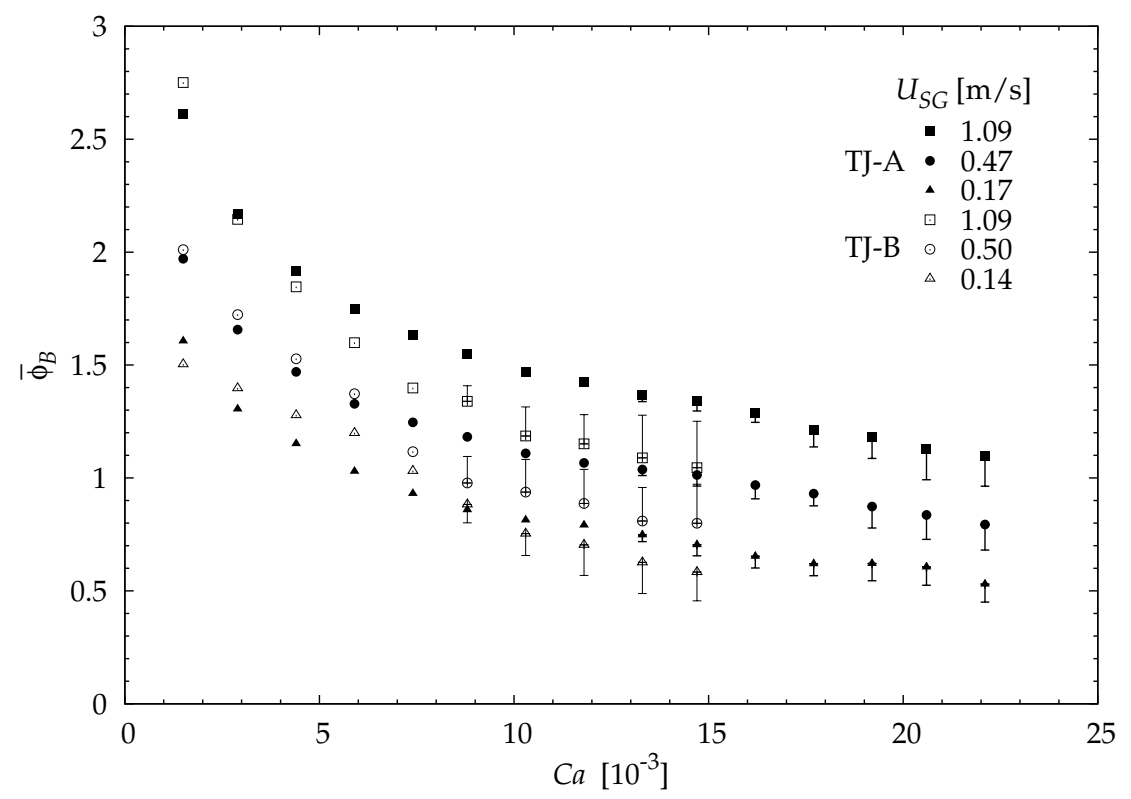

Fig. 4 Normalized bubble equivalent diameter as a function of the capillary number for several gas superficial velocities. Solid points: TJ-A; empty points: TJ-B.

$10^{-3}$ (TJ-A) and $8.8 \cdot 10^{-3}$ (TJ-B). To avoid the overlap of some error bars, only one of the two error bars corresponding to each experimental point (the superior or the inferior bar) was plotted. Therefore, it should be noted in fig. 4 that the complementary (and missing) error bar of each point also exists, although it was removed from the graph in the interest of clarity.

Fig. 4 shows how $\bar{\phi}_{B}$ decreases with the increase of $C a$, as expected. This fact corresponds to the decrease of the bubble volume with $\mathrm{Ca}$ and it was previously observed in figs. 2-3. For a given value of the capillary number, $\bar{\phi}_{B}$ increases with the increase of the gas superficial velocity, which is also an expected result. When comparing two similar values of $U_{S G}$ in fig. 4, and depending on the value of the capillary number, the TJ-B configuration can produce either bigger or smaller bubbles than those provided by TJ-A. For example, when comparing $U_{S G}=0.47$ $\mathrm{m} / \mathrm{s}(\mathrm{TJ}-\mathrm{A})$ and $U_{S G}=0.5 \mathrm{~m} / \mathrm{s}$ (TJ-B), TJ-B produces bigger bubbles than TJ-A at small capillary numbers, but smaller ones at larger values of $C a$. Subsections 3.3 and 3.4 discuss in detail the bubble generation process and the resulting bubble size.

The polydispersity index $\left(I_{p}\right)$ is used to quantify the effect of the gas injection method on the regularity of the bubble generation process. The polydispersity index is defined here as $I_{p}=\left(S D / \phi_{B}\right) \cdot 100 \%$, being $S D$ the standard deviation of the bubble equivalent diameter. Thus, the bubble size variability is studied as a relative change (concerning $\phi_{B}$ ), instead of as an absolute change.

Fig. 5 shows how $I_{p}$ evolves as the capillary number increases. The polydispersity index behaves (concerning $\mathrm{Ca}$ ) similarly for all the values of $U_{S G}$ analyzed in this work. For the sake of clarity, only three values of the gas superficial velocity 
(same values than plotted in fig. 4) are shown in the figure for each gas injection configuration. Up to $C a=7.4 \cdot 10^{-3}$, all experimental data collapse around the value $I_{p}=0.9 \%$ (i.e. the average value in this region). Separately analyzed, the average value of $I_{p}$ below $C a=7.4 \cdot 10^{-3}$ is $0.8 \%$ and $1 \%$ for TJ-A and TJ-B, respectively. These small values are in good agreement with values found in the related literature (Garstecki et al., 2005; Xu et al., 2006; Christopher et al., 2008; Zhao and Middelberg, 2011; Zhang et al., 2014; Mahdia et al., 2017).

Concerning the regularity of the bubble generation process, there does not seem to be any significant difference between the two methods when $C a \leq 7.4 \cdot 10^{-3}$, and highly monodisperse bubbles can thus be obtained with both configurations. However, $I_{p}$ considerably grows from value $C a=8.8 \cdot 10^{-3}$ in the configuration TJ-B. Beyond $\mathrm{Ca}=10.3 \cdot 10^{-3}, I_{p}$ is in fact greater than $10 \%$ in all cases of that configuration.

The TJ-A configuration forms monodisperse bubbles at higher capillary numbers than TJ-B. Up to $C a=11.8 \cdot 10^{-3}$, the polydispersity index remains below $2 \%$, with a mean value of $1.1 \%$. Beyond this value, $I_{p}$ begins to increase, but at a slower pace than observed in TJ-B. It is not until the value $C a=20.6 \cdot 10^{-3}$ that TJ-A reaches the same dispersion level, grosso modo, as the observed in TJ-B from value $C a=10.3 \cdot 10^{-3}$. Hence, the limits of monodispersity, as far as the capillary number is concerned, are determined by the values $C a=11.8 \cdot 10^{-3}$ (TJ-A) and $C a=7.4 \cdot 10^{-3}$ (TJ-B).

Fig. 5 also suggests that for a given $C a, I_{p}$ tends to decrease when increasing $U_{S G}$. For example, note that $I_{p}$ values are smaller for $1.09 \mathrm{~m} / \mathrm{s}$ than for $0.17 \mathrm{~m} / \mathrm{s}$ (TJ-A) and $0.14 \mathrm{~m} / \mathrm{s}$ (TJ-B). The previous fig. 4 shows how the standard deviation of the normalized bubble equivalent diameter increases with the increase of the capillary number (both in TJ-A and TJ-B), while it does not seem to change significantly for a given $C a$ (considering TJ-A and TJ-B separately). Thus, the increase of $U_{S G}$ (for a given $C a$ and gas injection method) has a greater impact on the bubble equivalent diameter (which increases) than on its standard deviation, which causes the consequent decrease of $I_{p}$.

\subsection{Bubble generation frequency}

Figs. 6 and 7 show the bubble generation frequency as a function of the gas superficial velocity for TJ-A and TJ-B, respectively. In the interest of clarity, experimental data from TJ-A and TJ-B have been plotted separately but using the same scale values. Only values of the capillary number that provide monodisperse bubbles (in both methods simultaneously) are plotted in these figures, i.e. $C a \leq 7.4 \cdot 10^{-3}$ (see subsection 3.1). The standard deviation of the frequency has been calculated for each experimental value and plotted as error bars. Error bars smaller than the corresponding symbols have not been represented.

The TJ-A configuration analyzed in this work has already been studied and previously reported (Arias et al., 2009, 2010; Arias and González-Cinca, 2016). Concerning the bubble generation frequency, it was observed (in TJ-A) that at very low gas flow rates, the frequency follows a close-to-linear tendency, while for higher values, the frequency progressively curves until reaching a constant saturation value $f_{\text {sat }}$. Therefore, two main regimes arise during the bubble generation process, 


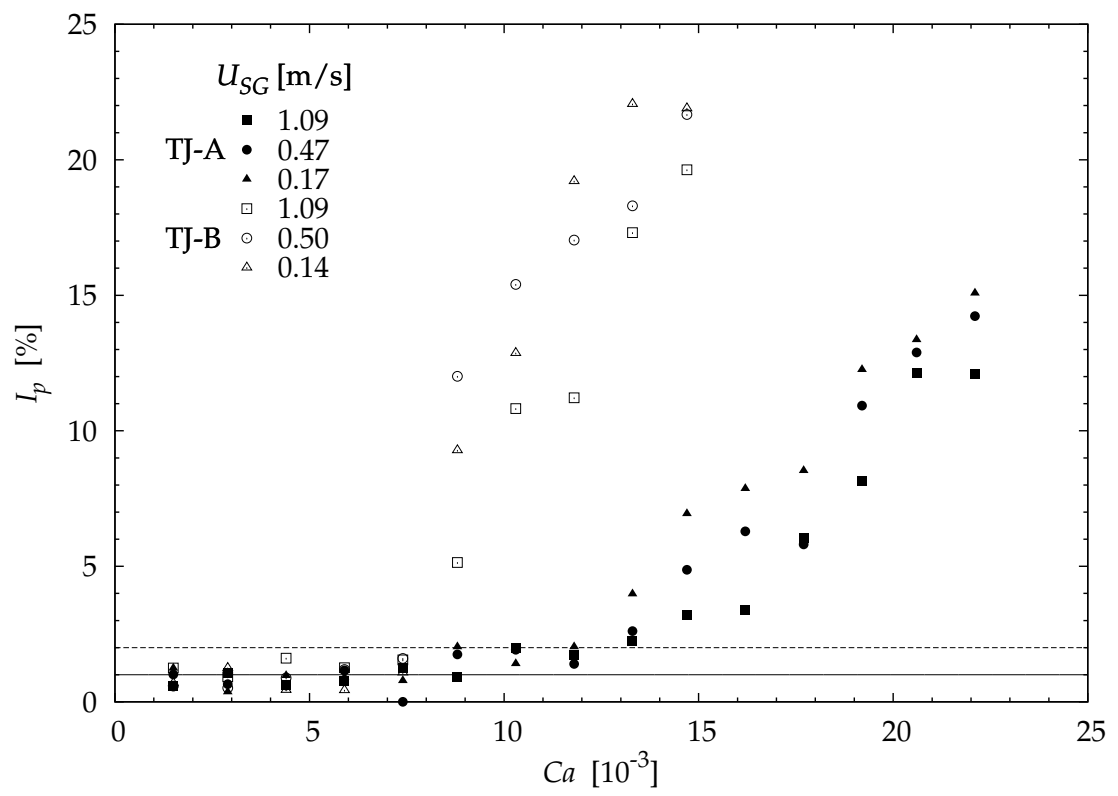

Fig. 5 Polydispersity index as a function of the capillary number for several gas superficial velocities. Solid points: TJ-A; empty points: TJ-B. Solid and dashed lines are guide for the eye corresponding to the $I_{p}$ values $1 \%$ and $2 \%$, respectively.

which were formerly referred to as the linear and the saturation regimes (Arias et al., 2009). Figs. 6 shows this expected behaviour for the TJ-A method.

New results show that TJ-B behaves in the same manner as TJ-A configuration, with a linear regime followed by a saturation regime for each $\mathrm{Ca}$ (see fig. 7). Since the gas injection strategy differs between these two configurations, this behaviour was not necessarily an expected result. The main difference between these two methods, though not the only one, seems to be that (on equal terms) TJ-A configuration achieves higher frequencies than TJ-B configuration in the linear regime, whereas in the saturation regime this trend is reversed and TJ-B reaches higher frequencies than TJ-A. In all the cases, the bubble generation frequency increases with the capillary number. These aspects will be analyzed in detail in subsections 3.3 and 3.4.

Arias and Montlaur (2017) proposed (for the TJ-A configuration) a simple fitting function aiming to smoothly connect the linear regime to the saturation region. It takes into account two fitting parameters, i.e. the initial slope of the linear regime $\left(a_{0}\right)$ and the value of the saturation frequency $\left(f_{\text {sat }}\right)$ :

$$
f=f_{\text {sat }}\left(1-e^{\left(-\frac{a_{0}}{f_{\text {sat }}} U_{S G}\right)}\right)
$$

While the first fitting parameter describes the linear behaviour of the linear regime $\left(f=a_{0} \cdot U_{S G}\right)$, the second one characterizes the saturation regime $(f=$ $\left.f_{\text {sat }}\right)$. Both parameters have an identified physical meaning. On one hand, the initial slope is related to the value of the minimum bubble volume that can be 


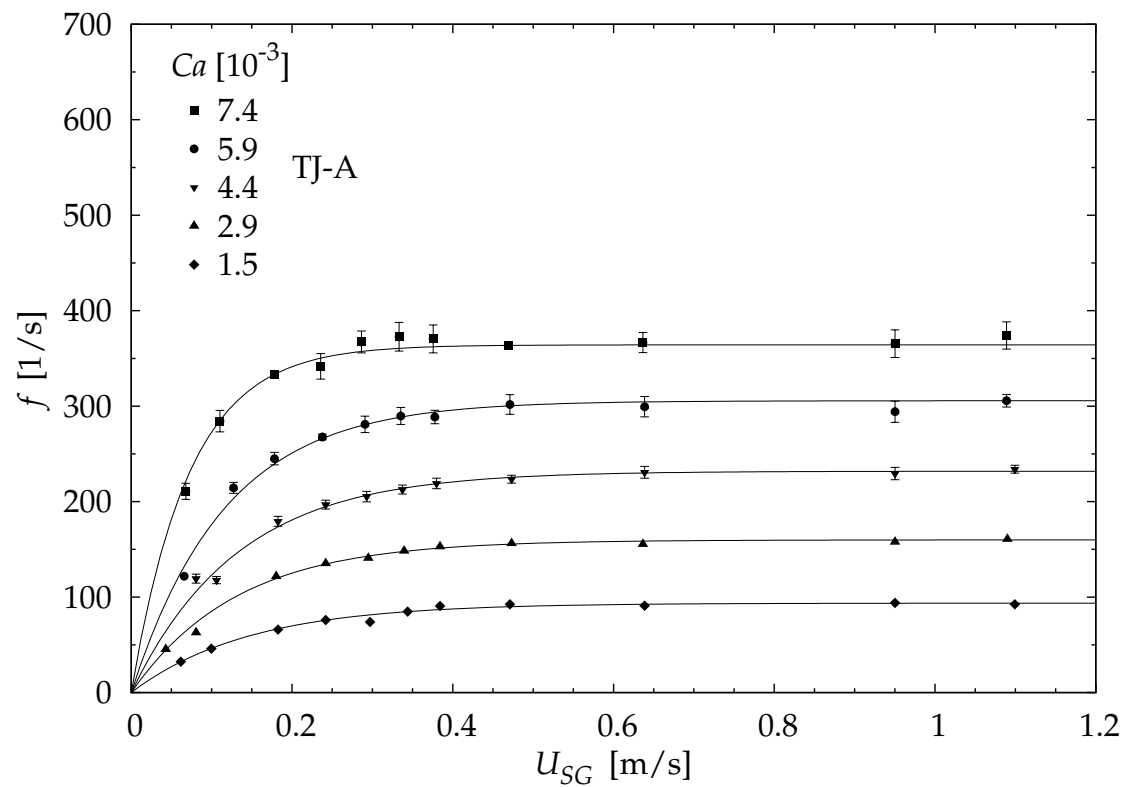

Fig. 6 TJ-A configuration: bubble generation frequency as a function of the gas superficial velocity for several capillary numbers. Lines correspond to eq. 2

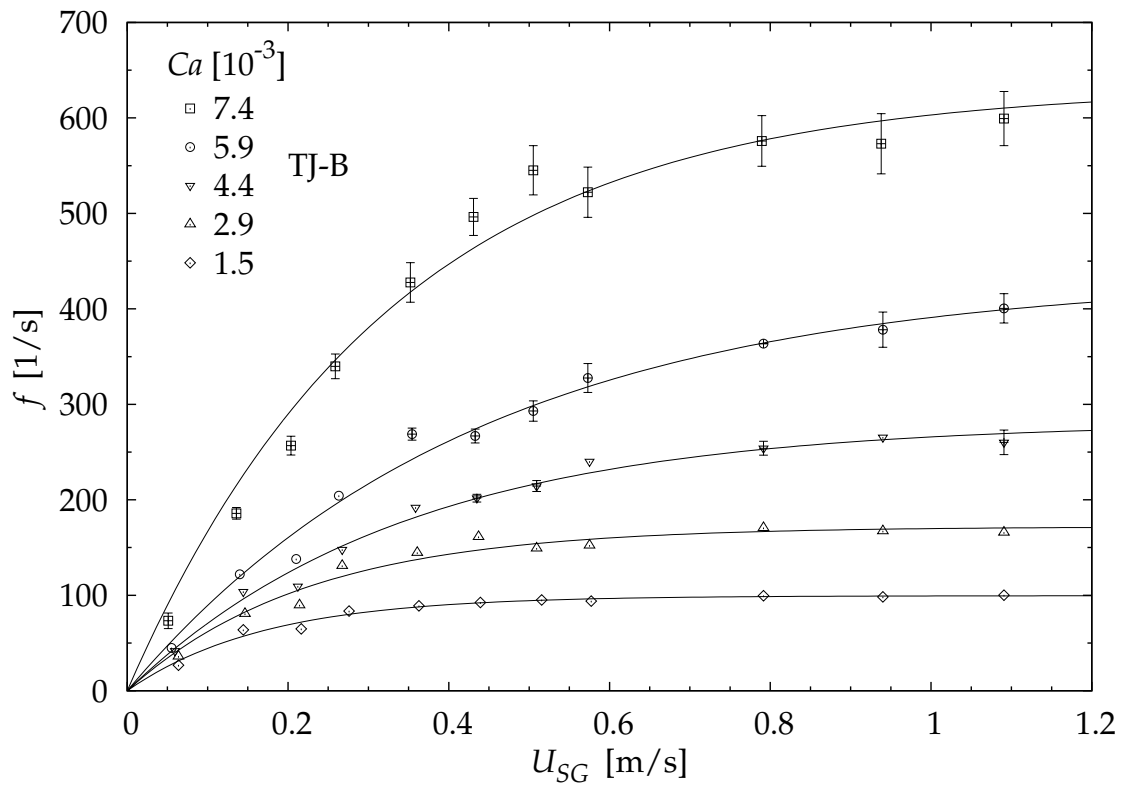

Fig. 7 TJ-B configuration: bubble generation frequency as a function of the gas superficial velocity for several capillary numbers. Lines correspond to eq. 2 


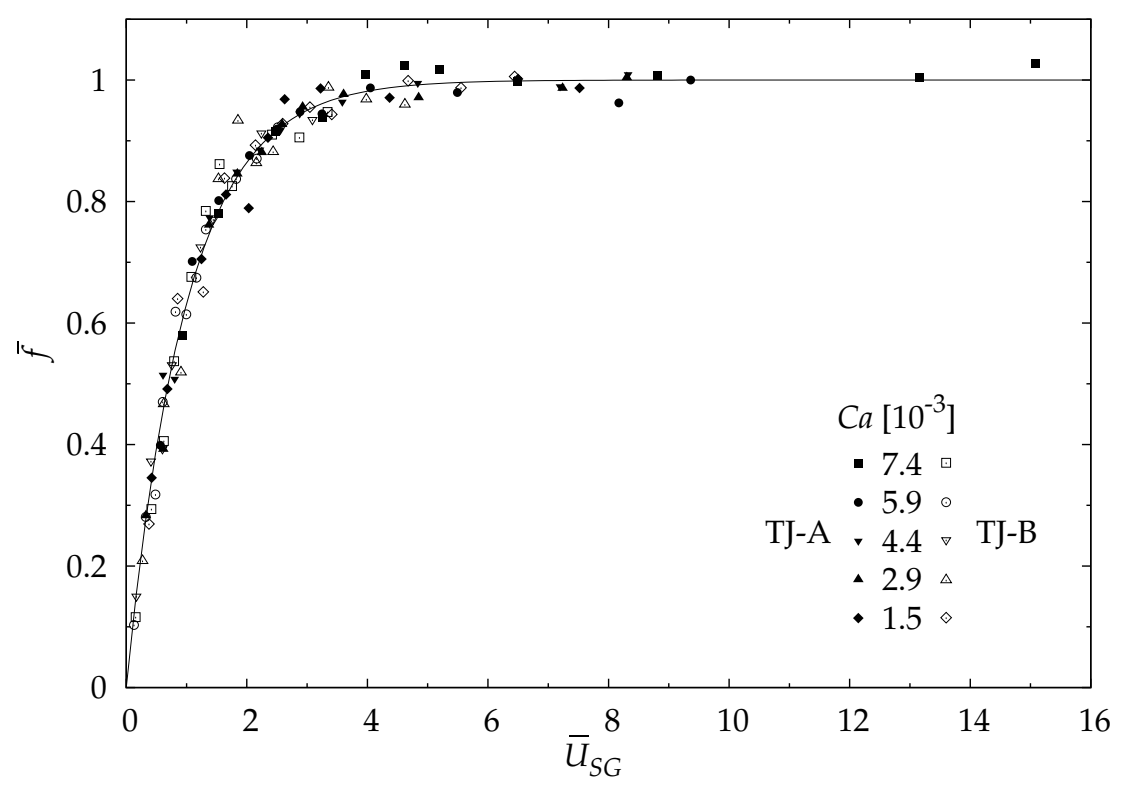

Fig. 8 Normalized frequency as a function of the normalized gas superficial velocity. Solid points: TJ-A; empty points: TJ-B. Line corresponds to eq. 3

generated for each capillary number, as follows: $\left.\bar{V}_{B}\right|_{\text {min }}=1 / \bar{a}_{0}$, where $\left.V_{B}\right|_{\text {min }}$ and $a_{0}$ have been normalized with $A \cdot \phi_{c}$ and $\phi_{c}$, respectively (Arias and Montlaur, 2018). On the other hand, the saturation frequency corresponds to the minimum time needed to form and detach a single bubble, again for a given capillary number (Arias et al., 2009).

Eq. 2 was used to fit the experimental data and subsequently plotted in figs. 67 (for each capillary number). Eq. 2 provided a good fit across the whole range of experimental data, for both TJ-A and TJ-B configurations. Fitting parameters $a_{0}$ and $f_{\text {sat }}$ will be analyzed in more detail in the following subsections. As a general remark, figs. 6 and 7 show that both parameters increase as $C a$ does, reducing, in consequence, the bubble volume, which is in good agreement with previous observations (Arias et al., 2010).

An alternative way to present all experimental data in a single graph arises when taking into account the crossover point $\left(U_{S G, C P}\right)$ between the linear and the saturation regimes. The crossover point can be calculated as the intersection of the two straight lines that characterize these regimes, i.e. $U_{S G, C P}=f_{\text {sat }} / a_{0}$. Normalizing $f$ with $f_{\text {sat }}$ and $U_{S G}$ with $U_{S G, C P}$, eq. 2 can be rewritten as

$$
\bar{f}=1-e^{-\bar{U}_{S G}}
$$

The same experimental values presented in figs. 6 and 7 have been correspondingly normalized and included in fig. 8. As shown by fig. 8, eq. 3 satisfactorily describes the behavior of the experimental data. 


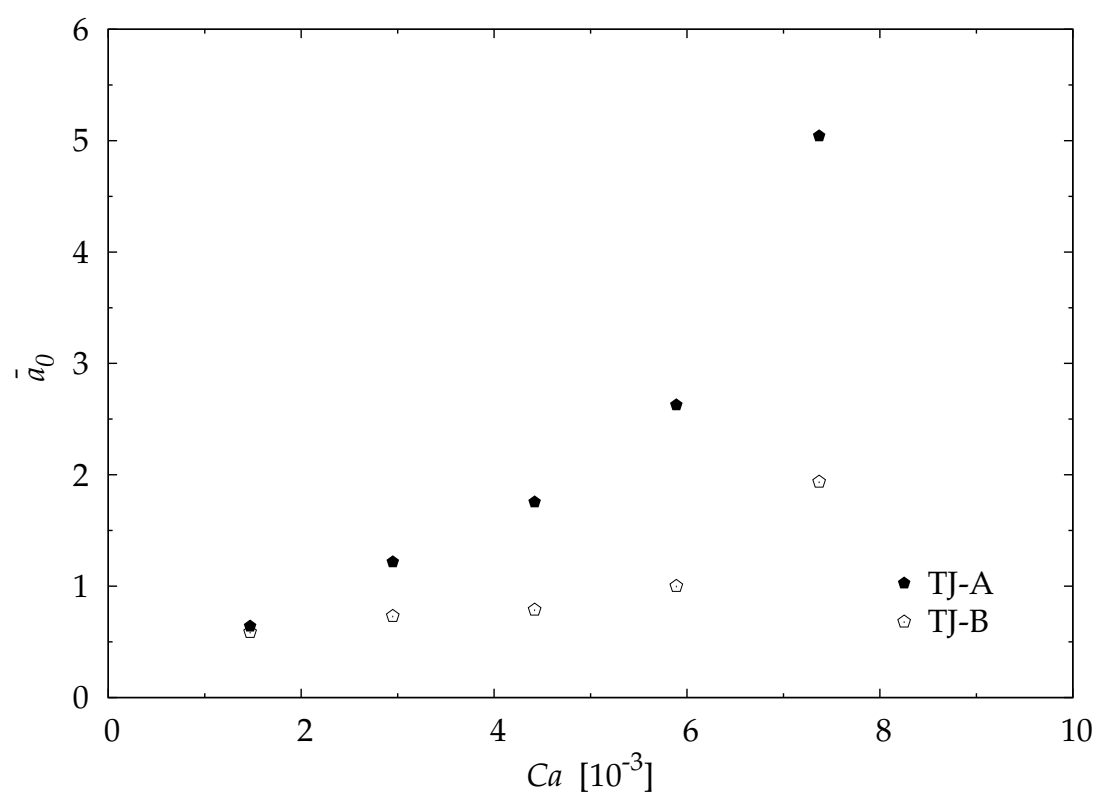

Fig. 9 Normalized initial slope as a function of the capillary number. Solid points: TJ-A; empty points: TJ-B

\subsection{Linear regime: $a_{0}$}

Fig. 9 shows the normalized initial slope as a function of the capillary number. The values of $a_{0}$ were obtained by fitting eq. 2 to experimental data. The initial slope $a_{0}$ was then normalized with the channel internal diameter. As shown in fig. 9, the normalized initial slope increases with $C a$, which is consistent with the fact that the bubble volume decreases with the increase of $C a$. Besides, the normalized initial slope is greater for TJ-A than for TJ-B, more as $C a$ increases. Thus, TJ-A achieves higher frequencies (and smaller bubbles) than TJ-B in the linear regime.

A better understanding of the linear regime can be gained by plotting $\left.\bar{V}_{B}\right|_{\text {min }}$ for each $C a$ (see fig. 10), where the normalized minimum bubble volume can be related to $a_{0}$ with the expression $\left.\bar{V}_{B}\right|_{\text {min }}=1 / \bar{a}_{0}$. Independently of the gas injection method (TJ-A or TJ-B), two linear trends appear in fig. 10. These trends have already been identified and reported in the related literature and correspond to the squeezing and dripping regimes (De Menech et al., 2008; Liu and Zhang, 2009). Both gas breakup mechanisms were briefly described at the beginning of section 3 . At low $C a$ (up to approximately $C a=4.4 \cdot 10^{-3}$ in fig. 10 ), $\left.\bar{V}_{B}\right|_{\text {min }}$ is not so much affected by the capillary number. Thus, liquid shearing forces does not seem to have significant influence in the bubble generation process (although the bubble volume decreases with $C a$ in any case). This behavior corresponds to the squeezing mechanism. For moderate $C a$ (beyond the value $C a=5.9 \cdot 10^{-3}$, approximately), $\left.\bar{V}_{B}\right|_{\text {min }}$ decreases sharply, which suggests that the liquid shearing forces begin to play a predominant role, activating the dripping mechanism. Therefore, fig. 10 
suggests that the squeezing-to-dripping transition takes place at a capillary number within the range $4.4-5.9 \cdot 10^{-3}$.

The related literature provides a large variety of values of the capillary number for the squeezing-to-dripping transition. The range $C a=4.4-5.9 \cdot 10^{-3}$ is consistent (same order of magnitude, small quantitative difference) with the previously reported observations of Xu et al. (2008) $\left(C a \sim 2 \cdot 10^{-3}\right)$, Oishi et al. (2009) $\left(C a \sim 3 \cdot 10^{-3}\right)$ and Guo and Chen $(2009)\left(C a \sim 5.8 \cdot 10^{-3}\right)$, whereas in other cases the quantitative difference can be of one order of magnitude, as in the works of De Menech et al. (2008) $\left(C a \sim 1.5 \cdot 10^{-2}\right)$ and Abate et al. $(2012)\left(C a \sim 7 \cdot 10^{-2}\right)$. We consider that the disagreement with the latter cases can be mainly due, in addition to the different approaches followed to detect the squeezing-to-dripping transition, to the fact that those studies were carried out in diverse conditions than the ones of the present work. For example, De Menech et al. (2008) and Abate et al. (2012) used T-junctions with a rectangular (or square) cross-section, instead of the circular one of this work. Moreover, liquid-liquid two-phase flows were used in those studies for generating droplets, in contrast with the gas-liquid two-phase flows used here for generating bubbles. The range $C a=4.4-5.9 \cdot 10^{-3}$ remains thus associated with the particular type of T-junction and conditions studied in this work.

The same two linear trends are observed in TJ-A and TJ-B and, consequently, fig. 10 reveals that the squeezing and dripping regimes are present in both configurations. However, TJ-A produces smaller bubbles than TJ-B in the range of capillary numbers analyzed in this work. Thus, TJ-A reaches higher frequencies than TJ-B in the linear regime, as stated before. A closer observation of the bubble formation cycle (in the squeezing and dripping regimes, separately) can provide useful information on this regard.

Fig. 11 shows an example of the complete bubble formation cycle in the squeezing regime $\left(C a=2.9 \cdot 10^{-3}\right)$. Time (in milliseconds) is indicated in each picture. To capture more relevant details of the bubble formation process, non-equispaced times are presented in the figure. At the beginning of the process, the emerging gas tends to fill the main channel (in the figure, from the initial instant up to approx. $13.5 \mathrm{~ms}$ (TJ-A) and $20 \mathrm{~ms}$ (TJ-B)), blocking it (see $15.3 \mathrm{~ms}$ (TJ-A) and $24.5 \mathrm{~ms}(\mathrm{TJ}-\mathrm{B}))$. This blockage causes a pressure buildup upstream the forming bubble. The liquid pushes the bubble downstream (approx. during the intervals 15.3-16.3 ms (TJ-A) and 24.5-27.8 ms (TJ-B)), provoking the gas breakup and the formation of a new bubble (at $16.8 \mathrm{~ms}$ (TJ-A) and $28.3 \mathrm{~ms}$ (TJ-B)).

During the squeezing process, the liquid also enters the gas channel, with greater penetration in the case of TJ-B than in TJ-A, which is a direct consequence of the gas injection method itself. In TJ-A, the liquid already flows parallel to the main channel and tends to stay flowing in this channel (although it also penetrates a little into the side channel). This scenario differs from configuration TJ-B, where the liquid enters the main channel perpendicularly. The liquid tends thus to flow simultaneously downstream and upstream the T-junction (entering the gas channel), obstructing the gas from flowing into the main channel. As a result, the gas takes longer (in TJ-B) to block again the main channel. For example, note in fig. 11 (for TJ-B) how the gas has not yet filled the T-junction crossing zone at $10 \mathrm{~ms}$, whereas for TJ-A the gas has almost blocked the main channel at $11.9 \mathrm{~ms}$. The start of a new bubble formation cycle is then retarded in TJ-B, which produces bigger bubbles while reaching lower bubble generation frequencies. As 


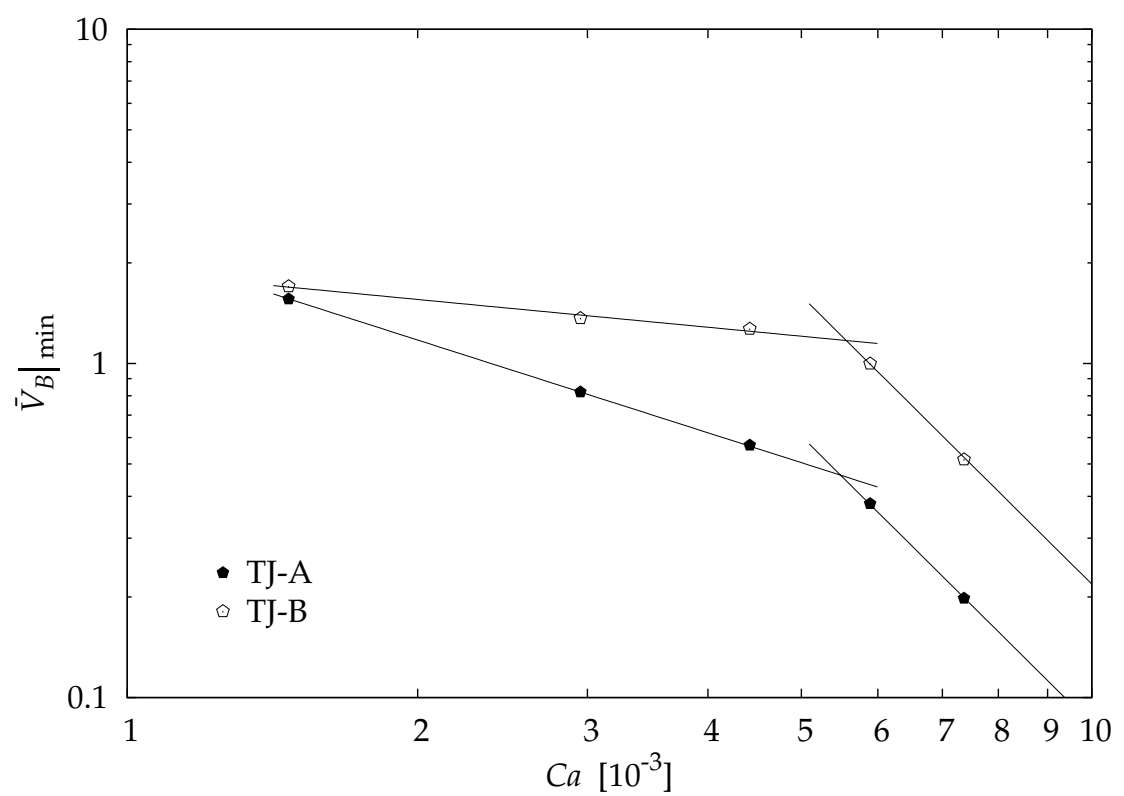

Fig. 10 Normalized minimum bubble volume as a function of the capillary number. Solid points: TJ-A; empty points: TJ-B. Lines are guide to the eye

the time required to form a bubble increases, so does the volume of the injected liquid, and the separation between consecutive bubbles also increases (see fig. 11).

Fig. 12 shows an example of the complete bubble formation cycle in the dripping regime $\left(\mathrm{Ca}=7.4 \cdot 10^{-3}\right)$. Time (in milliseconds) is again indicated in each picture. Unlike the squeezing regime, the liquid prevents now the gas from fully blocking the main channel (see for example $2.8 \mathrm{~ms}$ (TJ-A) and $12 \mathrm{~ms}$ (TJ-B)). The liquid flows through the existing gap between the forming bubble and the wall of the main channel (see for example $3.5 \mathrm{~ms}$ (TJ-A) and $13 \mathrm{~ms}$ (TJ-B)). As a result, the pressure buildup upstream the bubble decays. The increasing effect of the liquid shearing forces drags the gas downstream, reducing the bubble size. Just like in the squeezing regime, the liquid enters more deeply into the gas channel in TJ-B than in TJ-A, and more time is again required to inject the gas into the main channel and to form a bubble. Note for example how the gas begins to enter the T-junction cross-section at $9.8 \mathrm{~ms}$ (TJ-B), while only $2 \mathrm{~ms}$ are needed for TJ-A to be in analogous circumstances. This results, again, in higher bubble volumes and lower frequencies in TJ-B than in TJ-A.

\subsection{Saturation regime: $f_{\text {sat }}$}

Fig. 13 shows the saturation frequency as a function of the capillary number. The values of $f_{\text {sat }}$ were obtained by fitting eq. 2 to experimental data. Similar to observed in $a_{0}, f_{\text {sat }}$ increases with $C a$ in both configurations, which is again consistent with the fact that the bubble volume decreases with the increase of $C a$. 


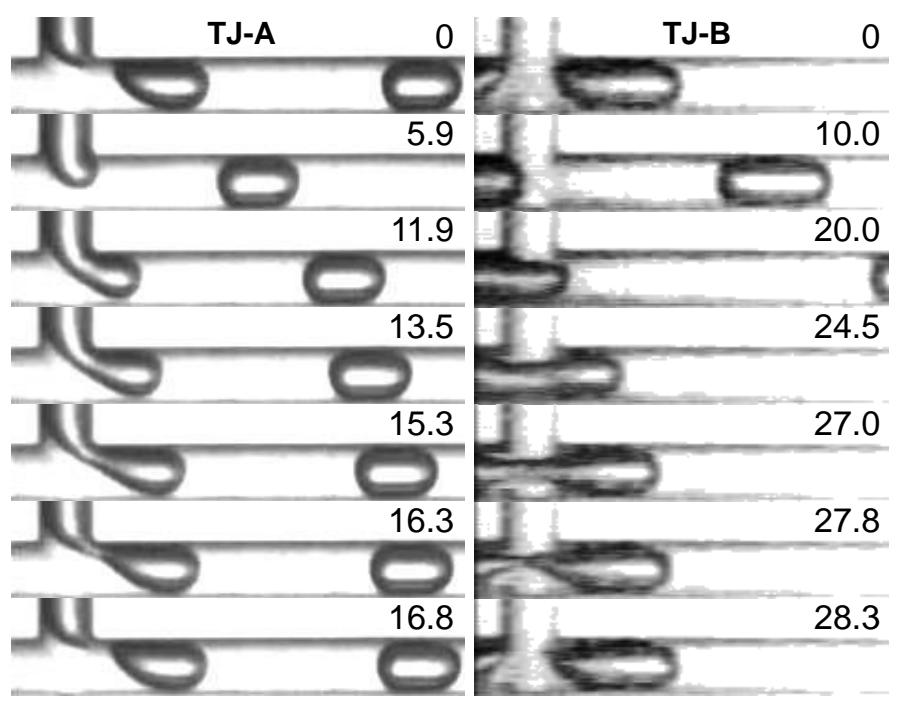

Fig. 11 Linear regime: bubble formation cycle in the squeezing regime. $C a=2.9 \cdot 10^{-3}$, $U_{S G} \approx 0.072 \mathrm{~m} / \mathrm{s}$

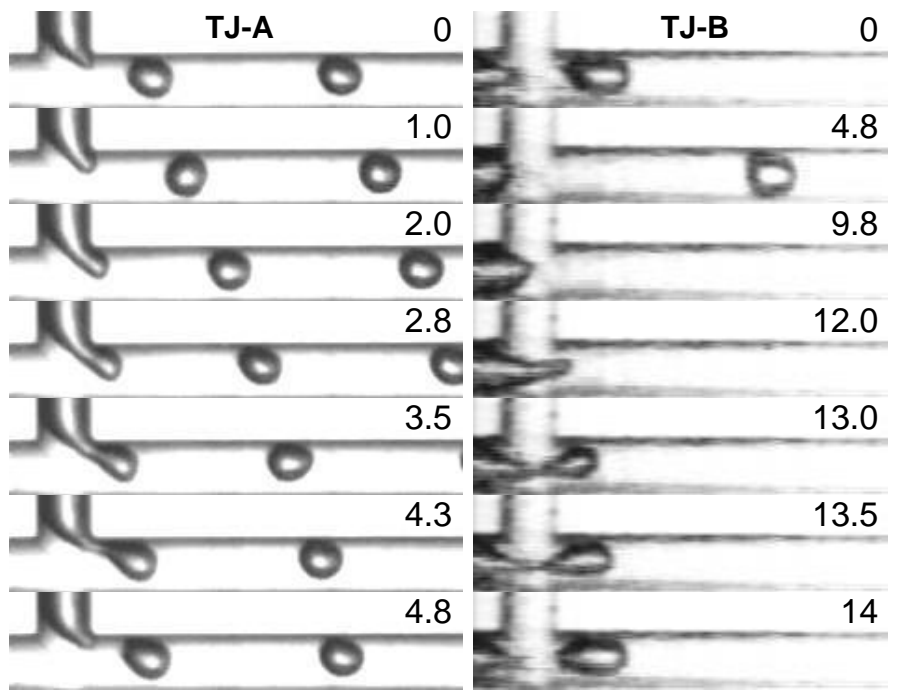

Fig. 12 Linear regime: bubble formation cycle in the dripping regime. $C a=7.4 \cdot 10^{-3}$, $U_{S G} \approx 0.059 \mathrm{~m} / \mathrm{s}$

In particular, TJ-B achieves higher values of frequency than TJ-A in the saturation regime (contrary to what occurred in the linear region), producing thus more and smaller bubbles. This fact becomes more evident as the capillary number increases. For example, at $\mathrm{Ca}=7.4 \cdot 10^{-3}$ the saturation frequency reaches the values 364 $\mathrm{Hz}$ (TJ-A) and $633 \mathrm{~Hz}$ (TJ-B), respectively.

Fig. 14 shows the formation of a bubble in the saturation regime. Time (in milliseconds) is indicated in each picture. The main change for the TJ-B configu- 


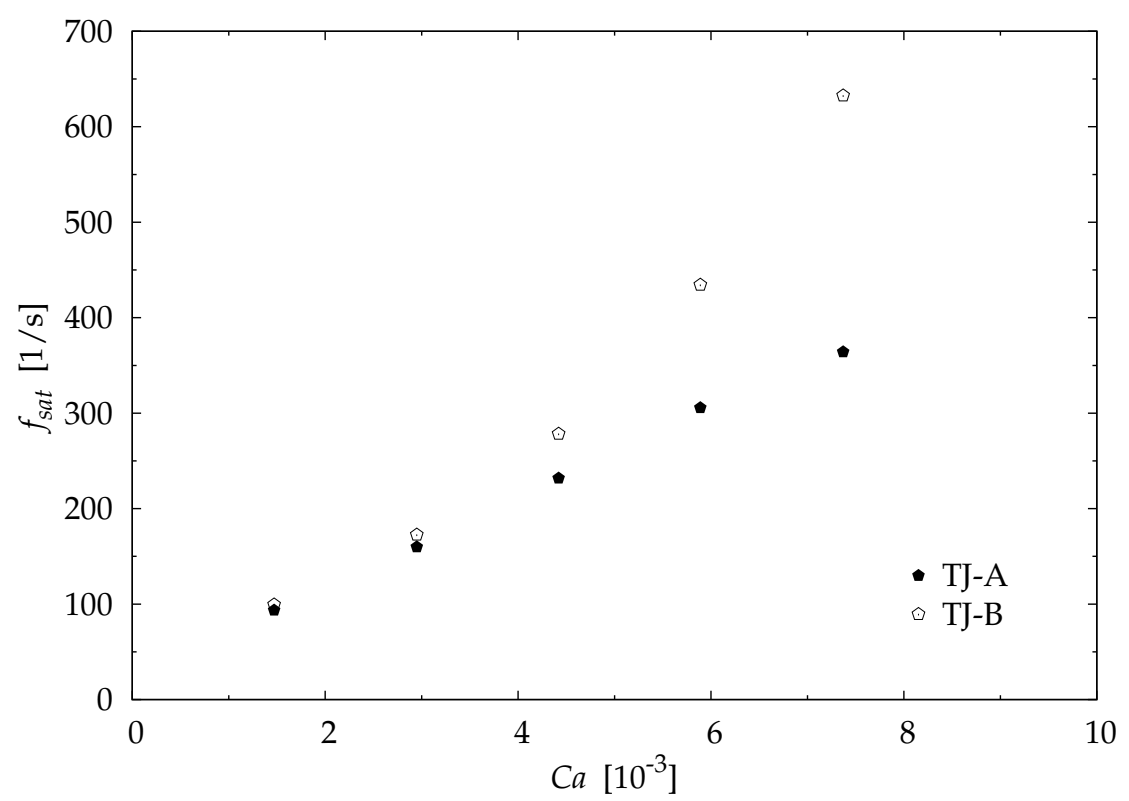

Fig. 13 Saturation frequency as a function of the capillary number. Solid points: TJ-A; empty points: TJ-B

ration (in comparison to figs. 11 and 12) is that the gas phase does not retract after the formation of a bubble. Just after the gas breakup (see for example $2.5 \mathrm{~ms}$ in TJ-B) the gas is already inside the T-junction crossing zone, ready to be dragged again by the liquid. This new situation (in comparison with the linear regime) reduces the bubble formation time in TJ-B. TJ-B configuration produces bubbles at a higher rate than TJ-A too, i.e. the gas breakup takes less time in TJ-B than in TJ-A. Therefore, the forces exerted by the continuous phase when it enters the main channel perpendicularly (TJ-B) are more effective (for producing bubbles) than those induced by the liquid when flowing parallel to the main channel (TJA). In simple words, in the saturation regime, the TJ-B configuration behaves like a guillotine that cuts the gas more quickly than the TJ-A method, in which the liquid has to drag the gas before cutting it off.

\section{Conclusions}

This paper analyzed a T-junction bubble generator and compared two different methods of gas injection. The T-junction consisted in two cylindrical channels (the main and the side channels) that intersected perpendicularly. Both channels had the same internal diameter $(1 \mathrm{~mm})$. The Bond number was small enough to neglect the gravitational effects. Therefore, the results of this work could be applied to the field of microgravity. Air was firstly injected into the side channel (referred to as TJ-A). After that, the gas was injected into the main channel (TJ-B configuration). Distilled water was used in all cases as the continuous phase. The 


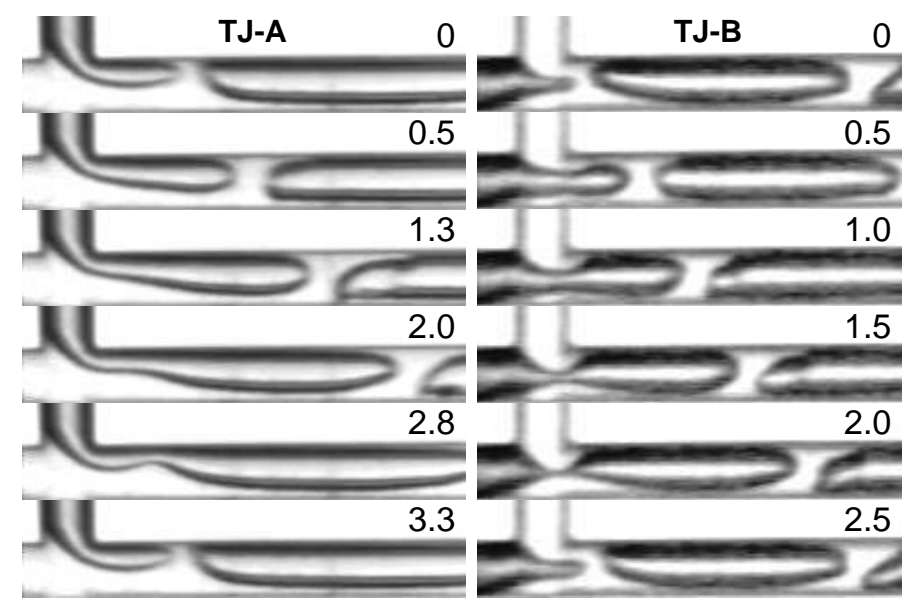

Fig. 14 Example of a complete bubble formation cycle in the saturation regime. $C a=5.9$. $10^{-3}, U_{S G} \approx 1.09 \mathrm{~m} / \mathrm{s}$

formation of bubbles was mainly controlled by the competition between interfacial and viscous forces. The capillary number became thus a key dimensionless number of this analysis.

The bubble formation process was observed to be affected by the gas injection method, as well as by the gas and liquid flow rates. In particular, the increase of the liquid superficial velocity (i.e. $\mathrm{Ca}$ ) had a significant impact on the regularity of the bubble generation process. The TJ-A method produced monodisperse bubbles at higher capillary numbers than configuration TJ-B. The monodispersity limits were found at $C a=11.8 \cdot 10^{-3}$ (TJ-A) and $C a=7.4 \cdot 10^{-3}$ (TJ-B). Below these limits, the average values of the polydispersity index were $1.1 \%$ (TJ-A) and $1 \%$ (TJ$\mathrm{B})$, and thus, highly monodisperse bubbles can be obtained with both methods. Beyond these limits, the bubble size dispersion increased, much more abruptly for TJ-B than for TJ-A.

The bubble generation frequency followed a close-to-linear tendency at very low gas flow rates, while it progressively curved until reaching a constant saturation value at higher gas flow rates. These two tendencies were referred to as linear (characterized by $a_{0}$ ) and saturation regimes (characterized by $f_{\text {sat }}$ ). The TJ-A configuration achieved higher frequencies than TJ-B in the linear regime, whereas TJ-B reached higher frequencies than TJ-A in the saturation regime. In all the cases, $a_{0}$ and $f_{\text {sat }}$ increased with the increase of $C a$. A new dimensionless expression of the bubble generation frequency was proposed. This expression provided an alternative way to predict the dimensionless bubble generation frequency.

For the range of $\mathrm{Ca}$ covered in this work, two types of gas breakup mechanisms were identified during the formation of bubbles (both in TJ-A and TJ-B): squeezing (at low $\mathrm{Ca}$ ) and dripping (at moderate $\mathrm{Ca}$ ) mechanisms. Both of them have been widely described in the related literature. In the linear regime, and regardless of the gas breakup mechanism, the liquid entered more deeply into the gas channel in TJ-B than in TJ-A, obstructing the gas from flowing into the main channel. Thus, the gas phase retracted more in TJ-B than in TJ-A, taking longer for the gas to flow again into the main channel. For such a reason, TJ-B required 
larger times to generate a bubble, which reduced the bubble generation frequency while increasing the bubble volume. This behaviour changed within the saturation regime, and configuration TJ-B became more effective than TJ-A in generating bubbles, reaching higher frequencies with smaller bubbles.

\section{References}

A.R. Abate, A. Poitzsch, Y. Hwang, J. Lee, J. Czerwinska, and D.A. Weitz. Impact of inlet channel geometry on microfluidic drop formation. Physical review E, 80: 026310-5, 2009. doi: 10.1103/PhysRevE.80.026310.

A.R. Abate, M. Pascaline, V. Van Steijn, and D.A. Weitza. Experimental validation of plugging during drop formation in a T-junction. Lab on a Chip, 12: 1516-1521, 2012. doi: 10.1039/C2LC21263C.

S. Arias and R. González-Cinca. Analysis of the characteristic lengths in the bubble and slug flow regimes generated in a capillary Tjunction. International Journal of Multiphase Flow, 87:167-174, 2016. doi: 10.1016/j.ijmultiphaseflow.2016.09.011.

S. Arias and A. Montlaur. Numerical study and experimental comparison of twophase flow generation in a T-junction. AIAA Journal, 55(5):1565-1574, 2017. doi: $10.2514 / 1 . J 055387$.

S. Arias and A. Montlaur. Influence of contact angle boundary condition on CFD simulation of T-junction. Microgravity Sci. Technol., 30(4):435-443, 2018. doi: $10.1007 / \mathrm{s} 12217-018-9605-\mathrm{x}$.

S. Arias, X. Ruiz, L. Ramírez-Piscina, J. Casademunt, and R. González-Cinca. Experimental study of a microchannel bubble injector for microgravity applications. Microgravity Sci. Technol., 21:107-118, 2009. doi: 10.1007/s12217-0089060-1.

S. Arias, R. González-Cinca, X. Ruiz, L. Ramírez-Piscina, and J. Casademunt. Characterization of the performance of a minibubble generator in conditions relevant to microgravity. Colloids Surfaces A: Physicochem Eng Aspects, 365: 52-57, 2010. doi: 10.1016/j.colsurfa.2010.03.004.

O. Carrier, D. Funfschilling, and H.Z. Li. Effect of the fluid injection configuration on droplet size in a microfluidic T junction. Physical review E, 89:013003-6, 2014. doi: 10.1103/physreve.89.013003.

G.F. Christopher, N.N. Noharuddin, J.A. Taylor, and S.L. Anna. Experimental observations of the squeezing-to-dripping transition in T-shaped microfluidic junctions. Physical review E, 78(3):036317, 2008. doi: 10.1103/PhysRevE.78.036317.

M. De Menech, P. Garstecki, F. Jousse, and H.A. Stone. Transition from squeezing to dripping in a microfluidic T-shaped junction. Journal of Fluid Mechanics, 595:141-161, 2008. doi: 10.1017/S002211200700910X.

T. Fu and Y. Ma. Bubble formation and breakup dynamics in microfluidic devices: A review. Chemical Engineering Science, 135:343-372, 2015. doi: 10.1016/j.ces.2015.02.016.

P. Garstecki, H.A. Stone, and G.M. Whitesides. Mechanism for flow-rate controlled breakup in confined geometries: A route to monodisperse emulsions. Physical review letters, 94:164501-4, 2005. doi: 10.1103/PhysRevLett.94.164501. 
P. Garstecki, M.J. Fuerstman, H.A. Stone, and G.M. Whitesides. Formation of droplets and bubbles in a microfluidic T-junction-scaling and mechanism of break-up. Lab on a Chip, 6:437-446, 2006. doi: 10.1039/b510841a.

F. Guo and B. Chen. Numerical study on taylor bubble formation in a microchannel T-junction using VOF method. Microgravity Sci. Technol, 21:51-58, 2009. doi: 10.1007/s12217-009-9146-4.

H. Liu and Y. Zhang. Droplet formation in a T-shaped microfluidic junction. Journal of Applied Physics, 106:034906, 2009. doi: 10.1063/1.3187831.

Y. Mahdia, K. Daoud, and L. Tadrist. Two-phase flow patterns and size distribution of droplets in a microfluidic t-junction: Experimental observations in the squeezing regime. Comptes Rendus Mecanique, 345:259-270, 2017. doi: 10.1016/j.crme.2017.02.001.

S. Malekzadeh and E. Roohi. Investigation of different droplet formation regimes in a T-junction microchannel using the VOF technique in OpenFOAM. Microgravity Sci. Technol., 27:231-243, 2015. doi: 10.1007/s12217-015-9440-2.

T. Nisisako, T. Torii, and T. Higuchi. Droplet formation in a microchannel network. Lab on a Chip, 2:24-26, 2002. doi: 10.1039/b108740c.

M. Oishi, H. Kinoshita, T. Fujii, and M. Oshima. Confocal micro-piv measurement of droplet formation in a t-shaped micro-junction. Journal of Physics: Conference Series, 147(1):012061, 2009. doi: 10.1088/1742-6596/147/1/012061.

K.S. Rezkallah. Weber number based flow-pattern maps for liquid-gas flows at microgravity. International Journal of Multiphase Flow, 22(6):1265-1270, 1996. doi: 10.1016/0301-9322(96)00047-X.

M. Suo and P. Griffith. Two-phase flow in capillary tubes. Journal of Basic Engineering, 86:576-582, 1964.

J. Tan, S.W. Li, K. Wang, and G.S. Luo. Gas-liquid flow in T-junction microfluidic devices with a new perpendicular rupturing flow route. Chemical engineering journal, 146 (3):428-433, 2009. doi: 10.1016/j.cej.2008.10.024.

T. Thorsen, R.W. Roberts, F.H. Arnold, and S.R. Quake. Dynamic pattern formation in a vesicle-generating microfluidic device. Physical Review Letters, 86 (18):4163-4167, 2001. doi: 10.1103/PhysRevLett.86.4163.

A.S. Utada, L.-Y. Chu, A. Fernandez-Nieves, D.R. Link, C. Holtze, and D.A. Weitz. Dripping, jetting, drops, and wetting: the magic of microfluidics. MRS Bulletin, 32(9):702-708, 2007. doi: 10.1557/mrs2007.145.

S. Van der Graaf, M.L.J. Steegmans, R.G.M. Van der Sman, C.G.P.H. Schroën, and R.M. Boom. Droplet formation in a T-shaped microchannel junction: A model system for membrane emulsification. Colloids and surfaces A, 266:106116, 2005. doi: 10.1016/j.colsurfa.2005.06.019.

J.H. Xu, S.W. Li, J. Tan, Y.J. Wang, and G.S. Luo. Controllable preparation of monodisperse $\mathrm{O} / \mathrm{W}$ and $\mathrm{W} / \mathrm{O}$ emulsions in the same microfluidic device. Langmuir, 22(19):7943-7946, 2006. doi: 10.1021/la0605743.

J.H. Xu, S.W. Li, J. Tan, and G.S. Luo. Correlations of droplet formation in Tjunction microfluidic devices: from squeezing to dripping. Microfluid Nanofluid, 5(6):711-717, 2008. doi: 10.1007/s10404-008-0306-4.

J.M. Zhang, E.Q. Li, and S.T. Thoroddsen. A co-flow-focusing monodisperse microbubble generator. J. Micromech. Microeng., 24:035008-6, 2014. doi: 10.1088/0960-1317/24/3/035008.

C.-X. Zhao and A.P.J. Middelberg. Two-phase microfluidic flows. Chemical Engineering Science, 66(7):1394-1411, 2011. doi: 10.1016/j.ces.2010.08.038. 